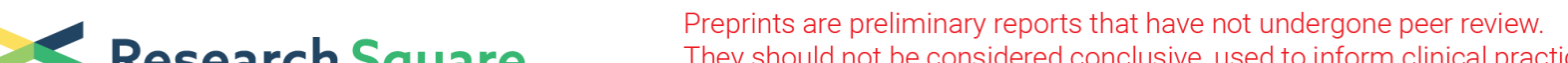 Research Square They should not be considered conclusive, used to inform clinical practice, or referenced by the media as validated information.
}

\section{Apatinib in Treating Clinical and Biochemical Recurrent Ovarian Cancer}

\section{Zhongyu Wang}

Third Military Medical University Second Affiliated Hospital: Xinqiao Hospital

\section{Yake Huang}

Third Military Medical University Second Affiliated Hospital: Xinqiao Hospital

\section{Ling Long}

Third Military Medical University Second Affiliated Hospital: Xinqiao Hospital

\section{Li Zhou}

Third Military Medical University Southwest Hospital

\section{Yan Huang}

Third Military Medical University Second Affiliated Hospital: Xinqiao Hospital

\section{Lei Gan}

Third Military Medical University Second Affiliated Hospital: Xinqiao Hospital

\section{Aimin Pu}

Third Military Medical University Second Affiliated Hospital: Xinqiao Hospital

\section{Sufen Li}

Third Military Medical University Second Affiliated Hospital: Xinqiao Hospital

\section{Rongkai Xie ( $D$ xierongkaixrk@163.com )}

Third Military Medical University Second Affiliated Hospital: Xinqiao Hospital https://orcid.org/00000002-6035-9192

\section{Research article}

Keywords: Ovarian cancer, Apatinib, Biochemical relapse, Anti-angiogenetic agents

Posted Date: October 6th, 2020

DOl: https://doi.org/10.21203/rs.3.rs-78764/v1

License: (c) (i) This work is licensed under a Creative Commons Attribution 4.0 International License. Read Full License 


\section{Abstract}

Background: Biochemical recurrence is defined according to the combined results of serum carbohydrate antigen and image; noteworthily, it generally precedes the onset of clinical evidence. Now treatment strategies of biochemical recurrence ovarian cancer (OC) remain controversial. Apatinib as monotherapy or in combination with other chemotherapeutic agents has shown its effect in the treatment of some advanced malignancies. In our study, we focused on the efficacy of apatinib in recurrent OC, especially its clinical activity in biochemical-only recurrent OC patients.

Methods: We retrospectively analyzed clinical material of 41 recurrent patients who had received apatinib monotherapy or apatinib plus chemotherapy between June 2016 and August 2018. Apatinib was administered at a $500 \mathrm{mg}$ daily dose. Response was determined according to measurable disease or serum carbohydrate antigen (CA)-125 levels. Progression-free survival (PFS) was estimated by KaplanMeier method.

Results: All patients were evaluable, 19 (46.34\%) had biochemical relapse and 22 (53.66\%) had clinical relapse. The objective response rate (ORR) and disease control rate (DCR) in the overall population were $31.71 \%$ and $78.05 \%$, respectively. The median PFS was 7 months (95\% confidence interval $5.43-8.57$ ). And in patients with biochemical-only relapse, the median PFS was 6 months, with ORR of $26.32 \%$ and DCR of $89.47 \%$.

Conclusions: Apatinib is a well-tolerated and effective agent to delay clinical progression of patients with biochemical-only recurrent OC. More important, our study shows the promising prospect for treating OC patients with asymptomatic biochemical relapse.

\section{Background}

Ovarian cancer (OC) is the first-leading cause of death due to gynecological malignancies [1]. Due to a lack of specific symptoms, nearly $75 \%$ of patients are diagnosed with advanced $\mathrm{OC}$ at the initial visit, contributing to the low 5-year survival rate (approximately 20\%) [2]. Cytoreductive surgery and platinumpaclitaxel combination chemotherapy are established as the primary treatments for advanced OC. However, the majority of patients who respond to initial treatment eventually experience a relapse and show low response to retreatment with cytotoxic therapy. Thus, the investigation of other effective treatment strategies remains a substantial clinical need.

Biochemical recurrence is defined as rising serum carbohydrate antigen (CA)-125 levels exceeding twice the upper limit of the normal range, without the disease being visualized on scans; noteworthily, it generally precedes the onset of clinical evidence by an average of 2 to 6 months [3,4]. In such cases, the choice between either a watch-and-wait policy or early therapeutic intervention remains controversial. Thus, it urgently needs a breakthrough to optimize therapy, to delay clinical disease progression to the extent that would require intravenous chemotherapy. 
Recently, the strategy of targeting angiogenesis has achieved success for the treatment of OC. Bevacizumab, a humanized monoclonal antibody that binds to all vascular endothelial growth factors (VEGF), has been approved by the European drug administration for the treatment of advanced ovarian carcinoma, specifically for recurrent platinum-sensitive or -resistant OC [5].

Apatinib is an oral vascular endothelial growth factor receptor 2 (VEGFR-2) inhibitor that inhibits tumor angiogenesis by blocking downstream signaling [6]. In China, apatinib has shown its effects in the thirdline treatment of advanced gastric adenocarcinoma and adenocarcinoma in the gastric-esophageal junction [7-9]. Some studies also suggested the use of apatinib in other advanced malignancies [10-17]. Therefore, in OC, apatinib has got increasing attention about its efficacy and safety [18-22]; however, to our knowledge, apatinib's efficacy in recurrent cancer, especially in biochemical-only recurrent $\mathrm{OC}$, is still unknown. Hence, we conducted this study to report the efficacy of apatinib in recurrent OC, and aimed to preliminarily assessing the outcome of apatinib monotherapy in biochemical recurrent $\mathrm{OC}$ patients.

\section{Methods}

\section{Patients}

In this retrospective study, we gathered the material of patients diagnosed with $\mathrm{OC}$ via pathologic evaluation, who had received apatinib monotherapy or treatment with apatinib plus chemotherapy between June 2016 and August 2018 in the first and second affiliated hospital of the Third Military Medical University. Patients were considered eligible for analysis if: 1) they received at least one line standard chemotherapy after debulking surgery and 2) relapse of disease was demonstrated by a measurable tumor or by an elevated level of CA-125. The study also enrolled patients who were intolerant to chemotherapy. Additional inclusion criteria included appropriate renal, hepatic, and hematopoietic function and an Eastern Cooperative Oncology Group performance status (ECOG PS) of 0-2. Patients with a history of bleeding, hypertension, ischemic cardiovascular disease, or proteinuria were ineligible for this study. The patients participating in this study provided written informed consent before study initiation.

\section{Treatments}

The administration of apatinib as monotherapy or in combination with chemotherapy was determined according to different patient needs. Apatinib monotherapy was applied to patients who were no longer tolerant to chemotherapy or patients with biochemical recurrence of disease. Patients with relapse of a measurable tumor received treatment with apatinib and chemotherapy based on a taxane or etoposide. The recommended initial dose of apatinib was $500 \mathrm{mg}$, po qd, half an hour after a meal at the same time every day. Chemotherapy was given simultaneously with apatinib for 28 days of one cycle. If intolerable toxicity occurred, the patient was informed to gradually reduce the dose to $250 \mathrm{mg}$ or discontinue the medication. 


\section{Evaluation}

The first evaluation for clinical efficacy and safety was performed at the end of the first cycle.

Subsequently, the interval of assessment changed to every two cycles. Treatment efficacy in patients with measurable disease was assessed by CT, MRI, and ultrasound scans. Complete remission (CR), partial remission (PR), stable disease (SD), and progressive disease (PD) were evaluated according to the Response Evaluation Criteria in Solid Tumors (RECIST) (Version 1.1). Responses of biochemical recurrent patients were determined through serum CA-125 levels. The CA-125 definition for response rate was based on the Rustin criteria [23]. A reduction of CA-125 to normalization that was maintained for at least 4 weeks was defined as CR, a $50 \%$ reduction as PR, a $25 \%$ increase as PD, and a situation beyond the above criteria was recognized as SD [24]. CR plus PR was categorized as objective response rate (ORR) and CR, PR, plus SD was defined as disease control rate (DCR). The period from initial treatment to disease progression or death was defined as progression-free survival (PFS). Drug-related adverse effects were evaluated and graded according to the National Cancer Institute Common Terminology Criteria for Adverse Events (NCl-CTCAE) (version 4.0).

\section{Statistical analysis}

The percentage method was used for categorical variables and drug safety analysis. PFS was analyzed by the Kaplan-Meier method, and the corresponding figures were drawn using GraphPad Prism 8.0 software (GraphPad Software Inc., San Diego, CA, USA). A P value $<0.05$ was regarded statistically significant.

\section{Results}

\section{Patient characteristics}

Between June 2016 and August 2018, a total of 41 advanced OC patients were enrolled in this study. The median age was 53 years (range, 36-67). Most patients $(33,80.48 \%)$ presented with stage IIIC (Federation International of Gynecology and Obstetrics FIGO) disease. The histological type included serous carcinoma (high grade $70.73 \%$; low grade $21.95 \%$ ), mucinous carcinoma (4.88\%), and endometrioid carcinoma (2.44\%). Of 41 patients, $14(34.15 \%)$ had optimal debulking surgery, whereas the remaining 27 (65.85\%) received suboptimal debulking surgery. All were tumor recurrent patients after previous therapy; of these, 19 (46.34\%) patients had biochemical recurrence and 22 (53.66\%) patients had a visible tumor. Among the included patients, 29 (70.73\%) had receivedBaseline patients' characteristics 1-2 lines of chemotherapy and $12(29.27 \%)$ had received 3-5 lines of treatment before participating. In this study, 13 of $41(31.71 \%)$ patients were treated with apatinib in combination with chemotherapy and 28 patients (68.69\%) received apatinib monotherapy. Most patients (75.61\%) had good Eastern Cooperative Oncology Group performance status (ECOG PS) (0), and patients with ECOG PS of 1 and 2 accounted for 
$14.63 \%$ and $9.76 \%$, respectively. Detailed baseline clinical characteristics of the patients are shown in Table 1. 
Table 1

Baseline patients' characteristics

\begin{tabular}{|c|c|}
\hline Characteristics & No. \% \\
\hline Median age(rang)(years) & $53(36-67)$ \\
\hline \multicolumn{2}{|l|}{ FIGO stage } \\
\hline IIIA & $1(2.44 \%)$ \\
\hline IIIB & $3(7.32 \%)$ \\
\hline IIIC & $33(80.48 \%)$ \\
\hline IV & $4(9.76 \%)$ \\
\hline \multicolumn{2}{|l|}{ ECOG PS } \\
\hline 0 & $31(75.61 \%)$ \\
\hline 1 & $6(14.63 \%)$ \\
\hline 2 & $4(9.76 \%)$ \\
\hline \multicolumn{2}{|l|}{ Histology Type } \\
\hline High-grade serous carcinoma & $29(70.73 \%)$ \\
\hline Low-grade serous carcinoma & $9(21.95 \%)$ \\
\hline Mucinous carcinoma & $2(4.88 \%)$ \\
\hline Endometrioid carcinoma & $1(2.44 \%)$ \\
\hline \multicolumn{2}{|l|}{ Debulking Surgery } \\
\hline Optimal & $14(34.15 \%)$ \\
\hline Suboptimal & $27(65.85 \%)$ \\
\hline \multicolumn{2}{|l|}{ Biochemical recurrence } \\
\hline Yes & $19(46.34 \%)$ \\
\hline No & $22(53.66 \%)$ \\
\hline \multicolumn{2}{|l|}{ Previous chemotherapy lines } \\
\hline $1-2$ & $29(70.73 \%)$ \\
\hline $3-5$ & $12(29.27 \%)$ \\
\hline \multicolumn{2}{|l|}{ Treatment regimen } \\
\hline Combined chemotherapy & $13(31.71 \%)$ \\
\hline Single drug & $28(68.29 \%)$ \\
\hline
\end{tabular}




\section{Efficacy}

During a follow up in July 2019, all patients were evaluated. Efficacy analysis indicated that none of the 41 patients achieved Complete remission (CR), 13 patients achieved partial remission (PR), 19 patients maintain stable disease (SD), and 9 patients had progressive disease (PD), resulting in an objective response rate (ORR) of $31.71 \%$ and a disease control rate (DCR) of $78.05 \%$ (Table 2). The median progression-free survival (PFS) was 7 months (95\% Cl 5.43-8.57, Fig. 1A). Among the patients with biochemical relapse, ORR and DCR were $26.32 \%$ and $89.47 \%$ respectively (Table 2), with median PFS of 6 months (95\% Cl 4.39-7.61, Fig. 1B).

Table 2

Treatment response

\begin{tabular}{|llll|}
\hline & $\begin{array}{l}\text { Imageological } \\
\text { response }\end{array}$ & $\begin{array}{l}\text { Biochemical } \\
\text { response }\end{array}$ & $\begin{array}{l}\text { Overall } \\
\text { response }\end{array}$ \\
\hline Complete response (CR) & 0 & 0 & 0 \\
\hline Partial response (PR) & $8(36.36)$ & $5(26.32)$ & $13(31.71)$ \\
\hline Stable disease (SD) & $7(31.82)$ & $12(63.16)$ & $19(46.34)$ \\
\hline Progressive disease (PD) & $7(31.82)$ & $2(10.53)$ & $9(21.95)$ \\
\hline Objective response rate (ORR) & $8(36.36)$ & $5(26.32)$ & $13(31.71)$ \\
\hline Disease control rate (DCR) & $15(68.18)$ & $17(89.47)$ & $32(78.05)$ \\
\hline
\end{tabular}

\section{Safety}

Adverse reactions were assessed and are summarized in Table 3 . In general, most patients were tolerant to apatinib without any grade 4 adverse events (AEs). The most common grade 1-3 AE was hand-foot syndrome (46.4\%). Other common AEs were mucositis (41.5\%), fatigue (39.0\%), anorexia (39.0\%), proteinuria (36.6\%), hypertension (34.1\%), and thrombocytopenia (26.8\%). 
Table 3

Adverse events

\begin{tabular}{|llllll|}
\hline Adverse events & \multicolumn{2}{l}{ Grades } & & & \\
\cline { 2 - 5 } & $\mathbf{1}$ & $\mathbf{2}$ & $\mathbf{3}$ & $\mathbf{4}(\mathbf{n}, \%)$ & Total (n, \%) \\
& $(\mathbf{n}, \%)$ & $\mathbf{( n , \% )}$ & $\mathbf{( n , \% )}$ & & \\
\hline Hand-foot syndrome & $5(12.2 \%)$ & $9(22.0 \%)$ & $5(12.2 \%)$ & 0 & $19(46.4 \%)$ \\
\hline Mucositis & $3(7.3 \%)$ & $6(14.6 \%)$ & $8(19.5 \%)$ & 0 & $17(41.5 \%)$ \\
\hline Anorexia & $12(29.3 \%)$ & $3(7.3 \%)$ & $1(2.4 \%)$ & 0 & $16(39.0 \%)$ \\
\hline Fatigue & $4(9.8 \%)$ & $7(17.1 \%)$ & $5(12.2 \%)$ & 0 & $16(39.0 \%)$ \\
\hline Pain & $6(14.6 \%)$ & $4(9.8 \%)$ & $1(2.4 \%)$ & 0 & $11(26.8 \%)$ \\
\hline Hypertension & $5(12.2 \%)$ & $6(14.6 \%)$ & $3(7.3 \%)$ & 0 & $14(34.1 \%)$ \\
\hline Proteinuria & $11(26.8 \%)$ & $4(9.8 \%)$ & 0 & 0 & $15(36.6 \%)$ \\
\hline Diarrhea & $1(2.4 \%)$ & $2(4.9 \%)$ & 0 & 0 & $3(7.3 \%)$ \\
\hline Transaminase increased & $1(2.4 \%)$ & $3(7.3 \%)$ & $1(2.4 \%)$ & 0 & $5(12.2 \%)$ \\
\hline Neutropenia & $2(4.9 \%)$ & $2(4.9 \%)$ & $1(2.4 \%)$ & 0 & $5(12.2 \%)$ \\
\hline Thrombocytopenia & $6(14.6 \%)$ & $3(7.3 \%)$ & $2(4.9 \%)$ & 0 & $11(26.8 \%)$ \\
\hline
\end{tabular}

\section{Discussion}

Traditional therapies for OC, including debulking surgery and chemotherapy, cannot yield a good response rate in all relapsed $\mathrm{OC}$ patients. Efforts to understand $\mathrm{OC}$ biology have facilitated the development of new targeted antineoplastic agents. In cancer, angiogenesis contributes to tumor growth and invasion [25]. Multiple growth factors play proangiogenic roles, including VEGF, epidermal growth factor (EGF), and platelet-derived growth factors (PDGF); of these, the VEGF pathway is pivotal in angiogenesis [26]. Bevacizumab, a monoclonal antibody targeting VEGF-A, has been approved for the treatment of recurrent platinum-sensitive or -resistant OC [5]. Several multitargeted receptor tyrosine kinase inhibitors (TKIs), such as imatinib, cediranib, sorafenib, sunitinib, and pazopanib, target VEGFR, PDGFR, and FGFR. Many of these inhibitors have been or are being evaluated in clinical trials in $\mathrm{OC}$, and some agents have exhibited inhibitory effects [5].

Most of the studies demonstrating clinical activity in advanced OC are small case reports. Only two prospective studies tested the efficacy of apatinib treatment in advanced OC. One is a single arm clinical study, which assessed the efficacy and safety of apatinib as monotherapy in patients with recurrent platinum-resistant epithelial OC [19]. The ORR and DCR in 28 patients receiving apatinib $500 \mathrm{mg}$ daily were $41.4 \%$ and $68.9 \%$, respectively, and the median PFS and OS were 5.1 months and 14.5 months [19]. The other study assessed the activity of apatinib plus etoposide in the treatment of patients with 
platinum-resistant or -refractory OC, showing an ORR of $54 \%$ and DCR of $86 \%$ [18]. The toxicity of apatinib in monotherapy and combined therapy were both manageable $[18,19]$. In our study, we found that, in the overall population, the median PFS was 7 months, and that the ORR was $31.71 \%$ and DCR was $78.05 \%$, supporting the clinical activity of apatinib in recurrent OC. The safety of apatinib at a dose of $500 \mathrm{mg}$ daily was similar to that reported in the above studies.

The National Comprehensive Cancer Network (NCCN) guidelines for OC suggest that biochemical recurrent patients may: 1 ) enroll in a clinical trial; 2 ) delay treatment until clinical relapse; 3 ) receive immediate platinum-based recurrence therapy; or 4) undergo best supportive care [27]. Thus, several studies aimed to investigate low-toxicity agents to delay the appearance of measurable disease in these patients [28]. However, no efficacy agent was confirmed until now. In our study, we demonstrated the efficacy of apatinib in biochemical recurrent OC patients with a median PFS of 6 months. This result implied that early treatment using apatinib in biochemical-only recurrent $\mathrm{OC}$ may extend time to clinical disease progression and delay time to intravenous chemotherapy, with low toxicity. However, a large sample study is needed to confirm the effect of apatinib in biochemical relapse.

One of the potential shortcomings of this study is that it was a relatively small-scale retrospective study; only 19 biochemical recurrent patients were evaluable. Prospective studies on a large sample cohort are needed to confirm the value of apatinib for the treatment of biochemical relapse patients. Additionally, this study failed to find a biomarker to predict the efficacy in biochemical recurrent patients. In this study, patients experienced similar grades of AEs to previous studies of apatinib treatment in OC. Hand-foot syndrome, mucositis, fatigue, anorexia, proteinuria, and hypertension were the most common adverse effects; however, all were tolerable.

\section{Conclusions}

In this study, we focused on the activity of apatinib in biochemical recurrent OC patients. Results indicate that apatinib might be promising for such patients to delay clinical disease progression and intravenous chemotherapy. More important, our study brought the potential breakthrough for treating OC patients with asymptomatic biochemical relapse.

\section{Abbreviations}

OC: Ovarian Cancer; CR: Complete Remission; PR: Partial Remission; SD: Stable Disease (SD); PD: Progressive Disease; ORR: Objective Response Rate; DCR: Disease Control Rate; PFS: Progression-Free Survival; AE: Adverse Event.

\section{Declarations}

\section{Ethics approval and consent to participate}


This retrospective study was approved by the institutional review board of Xinqiao Hospital, Third Military Medical University, China. The patients participating in this study provided written informed consent before study initiation.

\section{Consent for publication}

Not applicable.

\section{Availability of data and materials}

The datasets used and analyzed in the current study are available from the corresponding author upon reasonable request.

\section{Competing interests}

The authors have no conflicts of interest.

\section{Funding}

This work was supported by Scientific and Technological Innovation Program of Social Undertakings and People's Livelihood Guarantee of Chongqing (cstc2016shms-ztzx10004 to R.X.) and the National Natural Science Foundation of China (Number 81372271 to R.X., Number 81802865 to Z.W.).

\section{Authors' contributions}

Rongkai Xie designed the research study and revised the manuscript; Zhongyu Wang and Yake Huang performed statistical analysis and drafted the manuscript; Ling Long, Li Zhou, Yan Huang, Aiming Pu, and Sufen Li participated in the cases recruit and follow-up of this study; Lei Gan assisted in the statistical analysis. All authors read and approved the final manuscript.

\section{Acknowledgements}

The authors sincerely thank all the patients and their families for participating in this study, and other investigators involved in this research.

\section{References}

1. Siegel RL, Miller KD, Jemal A. Cancer statistics, 2019. CA Cancer J Clin. 2019;69(1):7-34. 
2. Martin LP, Schilder RJ. Management of recurrent ovarian carcinoma: current status and future directions. Semin Oncol. 2009;36(2):112-25.

3. Hising C, Anjegard IM, Einhorn N. Clinical relevance of the CA 125 assay in monitoring of ovarian cancer patients. Am J Clin Oncol. 1991;14(2):111-4.

4. Tuxen MK, Soletormos G, Dombernowsky P. Serum tumor marker CA 125 for monitoring ovarian cancer during follow-up. Scandinavian journal of clinical and laboratory investigation. 2002;62(3):177-88.

5. Choi HJ, Armaiz Pena GN, Pradeep S, Cho MS, Coleman RL, Sood AK. Anti-vascular therapies in ovarian cancer: moving beyond anti-VEGF approaches. Cancer Metastasis Rev. 2015;34(1):19-40.

6. Scott LJ. Apatinib: A Review in Advanced Gastric Cancer and Other Advanced Cancers. Drugs. 2018;78(7):747-58.

7. Aoyama T, Yoshikawa T. Targeted therapy: Apatinib - new third-line option for refractory gastric or GEJ cancer. Nature reviews Clinical oncology. 2016;13(5):268-70.

8. Li J, Qin S, Xu J, Guo W, Xiong J, Bai Y, et al. Apatinib for chemotherapy-refractory advanced metastatic gastric cancer: results from a randomized, placebo-controlled, parallel-arm, phase II trial. J Clin Oncol. 2013;31(26):3219-25.

9. Li J, Qin S, Xu J, Xiong J, Wu C, Bai Y, et al. Randomized, Double-Blind, Placebo-Controlled Phase III Trial of Apatinib in Patients With Chemotherapy-Refractory Advanced or Metastatic Adenocarcinoma of the Stomach or Gastroesophageal Junction. J Clin Oncol. 2016;34(13):1448-54.

10. Hu X, Cao J, Hu W, Wu C, Pan Y, Cai L, et al. Multicenter phase Il study of apatinib in non-triplenegative metastatic breast cancer. BMC cancer. 2014;14:820.

11. Hu X, Zhang J, Xu B, Jiang Z, Ragaz J, Tong Z, et al. Multicenter phase II study of apatinib, a novel VEGFR inhibitor in heavily pretreated patients with metastatic triple-negative breast cancer. Int $J$ Cancer. 2014;135(8):1961-9.

12. Zhen L, Jiali C, Yong F, Han X, Hongming P, Weidong H. The Efficacy and Safety of Apatinib Treatment for Patients with Unresectable or Relapsed Liver Cancer: a retrospective study. J Cancer. 2018;9(16):2773-7.

13. Luo H, Zhang L, Yang B, Feng Y, Xiong Y, Zhang S, et al. A randomized phase 2 trial of apatinib vs observation as maintenance treatment following first-line induction chemotherapy in extensivestage small cell lung cancer. Invest New Drugs. 2019.

14. Tang J, Li XY, Liang JB, Wu D, Peng L, Li X. Apatinib Plus Chemotherapy Shows Clinical Activity in Advanced NSCLC: A Retrospective Study. Oncology Research Featuring Preclinical and Clinical Cancer Therapeutics. 2019;27(6):635-41.

15. Chen X, Qiu T, Zhu Y, Sun J, Li P, Wang B, et al. A Single-Arm, Phase II Study of Apatinib in Refractory Metastatic Colorectal Cancer. The oncologist. 2019;24(7):883-e407.

16. Li F, Liao Z, Zhao J, Zhao G, Li X, Du X, et al. Efficacy and safety of Apatinib in stage IV sarcomas: experience of a major sarcoma center in China. Oncotarget. 2017;8(38):64471-80. 
17. Xie L, Xu J, Sun X, Tang X, Yan T, Yang R, et al. Apatinib for Advanced Osteosarcoma after Failure of Standard Multimodal Therapy: An Open Label Phase II Clinical Trial. The oncologist. 2019;24(7):e542-e50.

18. Lan C-Y, Wang Y, Xiong Y, Li J-D, Shen J-X, Li Y-F, et al. Apatinib combined with oral etoposide in patients with platinum-resistant or platinum-refractory ovarian cancer (AEROC): a phase 2, singlearm, prospective study. The Lancet Oncology. 2018;19(9):1239-46.

19. Miao M, Deng G, Luo S, Zhou J, Chen L, Yang J, et al. A phase II study of apatinib in patients with recurrent epithelial ovarian cancer. Gynecol Oncol. 2018;148(2):286-90.

20. Cheng Y, Zhang J, Geng H, Qin S, Hua H. Multiline treatment combining apatinib with toptecan for platinum-resistant recurrent ovarian cancer patients: a report of three cases. Onco Targets Ther. 2018;11:1989-95.

21. Deng L, Wang Y, Lu W, Liu Q, Wu J, Jin J. Apatinib treatment combined with chemotherapy for advanced epithelial ovarian cancer: a case report. Onco Targets Ther. 2017;10:1521-5.

22. Zhang M, Tian Z, Sun Y. Successful treatment of ovarian cancer with apatinib combined with chemotherapy: A case report. Medicine (Baltimore). 2017;96(45):e8570.

23. Rustin, G.J., Use of CA-125 to assess response to new agents in ovarian cancer trials. J Clin Oncol, 2003. 21(10 Suppl): p. 187s-193s.

24. Wright, J.D., et al., Bevacizumab combination therapy in recurrent, platinum-refractory, epithelial ovarian carcinoma: A retrospective analysis. Cancer, 2006. 107(1): p. 83-9.

25. Folkman, J., What is the evidence that tumors are angiogenesis dependent? J Natl Cancer Inst, 1990. 82(1): p. 4-6.

26. Ferrara, N. and R.S. Kerbel, Angiogenesis as a therapeutic target. Nature, 2005. 438(7070): p. 967-74.

27. Network, N.C.C., <NCCN clinical practice guidelines in oncology.Ovarian Cancer. Version 1.2019.pdf>.

28. Kristeleit, R., et al., A randomised, open-label, phase 2 study of the IDO1 inhibitor epacadostat (INCB024360) versus tamoxifen as therapy for biochemically recurrent (CA-125 relapse)-only epithelial ovarian cancer, primary peritoneal carcinoma, or fallopian tube cancer. Gynecol Oncol, 2017. 146(3): p. 484-490.

\section{Figures}


A

All Patients

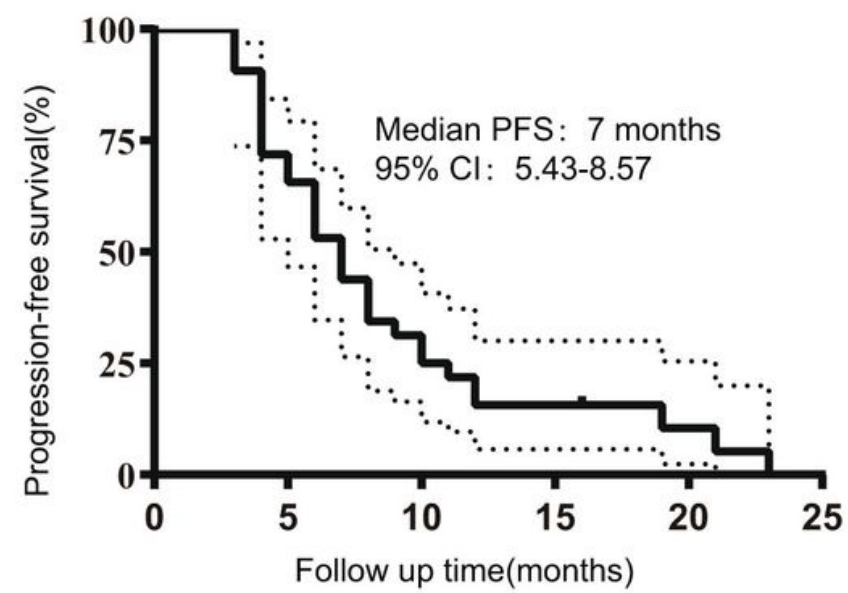

B

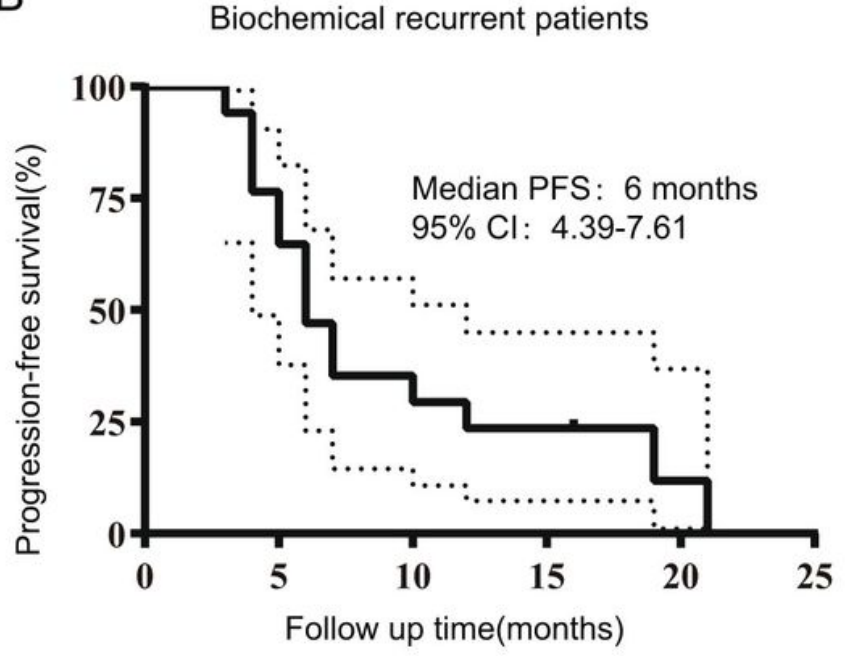

\section{Figure 1}

Kaplan-Meyer survival curve for estimated progression-free survival (PFS) in overall population (A) and in patients with biochemical relapse (B). 